\title{
Determination of Hydrogen Density by Swift Heavy Ions
}

\author{
Ge Xu, ${ }^{1,2,3,{ }^{*}}$ M. D. Barriga-Carrasco, ${ }^{4}$ A. Blazevic, ${ }^{5}$ B. Borovkov, ${ }^{6}$ D. Casas,${ }^{4}$ K. Cistakov, ${ }^{1}$ R. Gavrilin, ${ }^{6}$ M. Iberler, ${ }^{1}$ \\ J. Jacoby, ${ }^{1}$ G. Loisch, ${ }^{7}$ R. Morales ${ }^{4}$ R. Mäder, ${ }^{1}$ S.-X. Qin, ${ }^{8}$ T. Rienecker, ${ }^{1}$ O. Rosmej, ${ }^{5}$ S. Savin, ${ }^{6}$ A. Schönlein, ${ }^{1}$ \\ K. Weyrich, ${ }^{5}$ J. Wiechula, ${ }^{1}$ J. Wieser, ${ }^{9}$ G. Q. Xiao, ${ }^{2}$ and Y. T. Zhao ${ }^{10}$ \\ ${ }^{1}$ Institute of Applied Physics, Goethe University, 60438 Frankfurt am Main, Germany \\ ${ }^{2}$ Institute of Modern Physics, Chinese Academy of Sciences, Lanzhou 730000, People's Republic of China \\ ${ }^{3}$ University of Chinese Academy of Sciences, Beijing 100049, People's Republic of China \\ ${ }^{4}$ E.T.S.I. Industriales, Universidad de Castilla-La Mancha, E-13071 Ciudad Real, Spain \\ ${ }^{5}$ GSI Helmholtzzentrum für Schwerionenforschung GmbH, 64291 Darmstadt, Germany \\ ${ }^{6}$ Institute for Theoretical and Experimental Physics, 117218 Moscow, Russia \\ ${ }^{7}$ Deutsches Elektronen Synchrotron DESY, 15738 Zeuthen, Germany \\ ${ }^{8}$ Department of Physics, Chongqing University, Chongqing 401331, People's Republic of China \\ ${ }^{9}$ Excitech GmbH, 26419 Schortens, Germany \\ ${ }^{10}$ School of Science, Xi'an Jiaotong University, Xi'an 710049, People's Republic of China
}

(Received 16 May 2017; revised manuscript received 10 August 2017; published 15 November 2017)

\begin{abstract}
A novel method to determine the total hydrogen density and, accordingly, a precise plasma temperature in a lowly ionized hydrogen plasma is described. The key to the method is to analyze the energy loss of swift heavy ions interacting with the respective bound and free electrons of the plasma. A slowly developing and lowly ionized hydrogen theta-pinch plasma is prepared. A Boltzmann plot of the hydrogen Balmer series and the Stark broadening of the $H_{\beta}$ line preliminarily defines the plasma with a free electron density of $(1.9 \pm 0.1) \times 10^{16} \mathrm{~cm}^{-3}$ and a free electron temperature of $0.8-1.3 \mathrm{eV}$. The temperature uncertainty results in a wide hydrogen density, ranging from $2.3 \times 10^{16}$ to $7.8 \times 10^{18} \mathrm{~cm}^{-3}$. A $108 \mathrm{MHz}$ pulsed beam of ${ }^{48} \mathrm{Ca}^{10+}$ with a velocity of $3.652 \mathrm{MeV} / \mathrm{u}$ is used as a probe to measure the total energy loss of the beam ions. Subtracting the calculated energy loss due to free electrons, the energy loss due to bound electrons is obtained, which linearly depends on the bound electron density. The total hydrogen density is thus determined as $(1.9 \pm 0.7) \times 10^{17} \mathrm{~cm}^{-3}$, and the free electron temperature can be precisely derived as $1.01 \pm 0.04 \mathrm{eV}$. This method should prove useful in many studies, e.g., inertial confinement fusion or warm dense matter.
\end{abstract}

DOI: 10.1103/PhysRevLett.119.204801

In general, the free electron density and the free electron temperature are measured to describe a plasma. In practical application, however, the total particle density which governs, e.g., mean free path length or the state of matter, is of significant importance as well. For instance, the total particle density is a key factor for realizing metallic liquid hydrogen [1] and a critical condition for realizing the ignition of inertial confinement fusion [2-4], and, generally, the total particle density is used as a restricting condition for the definition of warm dense matter (WDM).

Although the total particle density can, in principle, be derived from the measured free electron density and temperature, the computation often involves large error margins due to the exponential dependence of the ionization degree on the free electron temperature for a lowionization plasma. Hence, we propose a novel method to extract directly the total particle density of a low-ionization hydrogen plasma by measuring the energy loss of a swift heavy ion beam interacting with the plasma column.

Beam-matter interactions are significant for many fields of research, such as accelerator physics, fusion plasma physics, high energy density physics, and astrophysics. The interaction of swift particles with matter, hence, has been extensively studied, resulting in early publications like the Bethe formula [5], stopping power data tables [6], and modern stopping power simulation programs like SRIM [7] or GEANT4 [8]. Moreover, the energy loss and stripping of heavy ions in a fully ionized (hydrogen) plasma have already been intensively investigated [9-15]. Taking advantage of these well-known beam-matter interactions, the total hydrogen density - and hence the ionization degreeof a lowly ionized hydrogen theta-pinch plasma has been measured during the cylindrical pinch phase. Furthermore, a highly precise free electron temperature can thus be derived in a self-consistent way.

In the experiments described here, a spherical theta pinch has been used as a plasma device, as described in Refs. [1624]. The energy stored in a capacitor bank is transferred to the target by a coil surrounding a 41 glass vessel filled with the working gas. Inside the glass vessel, the fast alternating magnetic field induces a strong electric field to ignite a discharge. The discharge then acts as a secondary winding to the primary copper coil, leading to a stable pinch compression along the discharge axis. In experiments 
described here, the total capacitance is summed as $37.5 \mu \mathrm{F}$, and the operation voltage is set at $14 \mathrm{kV}$. Before igniting the discharge, the initial pressure of the hydrogen gas inside the glass vessel is stabilized at $20 \mathrm{~Pa}$. Discharging the device, the hydrogen plasma first ignites near the wall of the glass vessel at a certain time; then dragging neutral particles with it, the hydrogen plasma moves inward to pinch; afterwards, the plasma dissipates outwardly, and hence one cycle of the dynamic movement of the plasma is completed. To determine the target conditions during the pinching, most of the diagnostics are carried out by spectroscopic methods. Emitted light is transported to the spectrometer by a quartz fiber optic cable. The $0.6 \mathrm{~m}$ spectrometer (Jobin-Yvon HRP) is equipped with an $1800 \mathrm{l} / \mathrm{mm}$ grating and blazed at $250 \mathrm{~nm}$. The spectra are recorded by an intensified and gated camera (Dicam Pro). By measuring the width of weak, nonbroadened impurity lines, an instrument resolution of $0.09 \mathrm{~nm}$ in full width at half maximum (FWHM) is determined. The spectra shown here are recorded at a fixed delay time $\Delta T$ after triggering the device, and the recording time is set at $1 \mu \mathrm{s}$. A relative spectrometerdetector intensity response function is determined, using a manufacturer-calibrated halogen-deuterium lamp combination (Heraeus Fiberlight).

In a first step, a preliminary electron temperature $T_{e}$ is obtained by a Boltzmann plot of the hydrogen Balmer lines $H_{\beta-\epsilon}$ using the system-response corrected spectra intensities. It should be noted that $H_{\alpha}$ has not been included in the plot because of the spectrally visible and strong centerline self-absorption. The Boltzmann plot results in a free electron temperature of between 0.8 and $1.3 \mathrm{eV}$.

In order to obtain the corresponding free electron density $n_{e}$, the Stark-broadened $H_{\beta}$ line has been analyzed, using tabulated FWHM values [25], as well as a peak-separation method [26], as shown in Fig. 1. Applying the tabulated value for a free electron temperature of $10 \mathrm{kK}$ in the free electron density range of $10^{16} \mathrm{~cm}^{-3}$ [25], the actual free

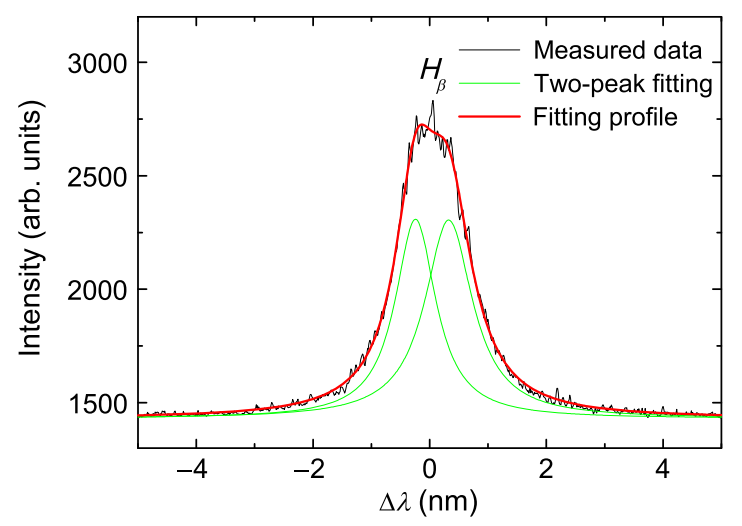

FIG. 1. The measured $H_{\beta}$ spectrum and a two-peak Lorenz fitting is shown. The measured peak separation of $0.57 \mathrm{~nm}$ results in a free electron density of $(1.9 \pm 0.1) \times 10^{16} \mathrm{~cm}^{-3}$ by using the two-peak separation method [26]. electron density is derived as $1.86 \times 10^{16} \mathrm{~cm}^{-3}$ from a measured FWHM of $1.47 \mathrm{~nm}$ in Fig. 1. However, because of a possible partial self-absorption of $H_{\beta}$, the obtained $n_{e}$ value might not be reliable without further information. Here, thus, a newly described method to determine $n_{e}$ has been used: To avoid analyzing a possibly modified width of the $H_{\beta}$ line, the peak separation within the line profile is used, as described in Ref. [26]. The peak separation in Fig. 1 is measured as $0.57 \mathrm{~nm}$, and the free electron density is hence determined as $(1.9 \pm 0.1) \times 10^{16} \mathrm{~cm}^{-3}$. It should be noted that the obtained density by the FWHM agrees fairly well with that by the two-peak separation method for the $H_{\beta}$ line. Hence, the self-absorption of $H_{\beta}$ for our plasma is negligible, and the free electron density is taken as $(1.9 \pm 0.1) \times 10^{16} \mathrm{~cm}^{-3}$ for further calculations.

Applying the measured free electron temperature of 0.8 $1.3 \mathrm{eV}$ and free electron density of $(1.9 \pm 0.1) \times 10^{16} \mathrm{~cm}^{-3}$, the total hydrogen density $n_{0}$ is obtained from a FLYCHK simulation [27,28], ranging between $2.3 \times 10^{16}$ and $7.8 \times 10^{18} \mathrm{~cm}^{-3}$. It should be emphasized that the moderate 20\%-30\% uncertainty of $T_{e}$ results in an uncertainty of more than 2 orders of magnitude for the total hydrogen number density $n_{0}$ due to the strong exponential dependence of the ionization degree on $T_{e}$.

Based on both the measured temperature and the estimated total hydrogen density, employing the dissociation equations in Ref. [29] and the simulation results shown in Ref. [30], one can conclude that the hydrogen molecules in the target are completely dissociated. Hence, the plasma target is composed of free electrons $e$, hydrogen ions $H^{1+}$, and monatomic hydrogen atoms $H^{0+}$.

To derive a precise value of the total hydrogen density in the plasma, beam-plasma interaction experiments are performed, and the energy loss as well as the charge-state distribution of the swift heavy ions is measured. For the experiments described here, the nuclear stopping is negligible compared to the electronic stopping. Hence, the energy loss is directly proportional only to the total electron density $n_{e}^{\text {total }}$ (equaling the total hydrogen density $n_{0}$ ), with $n_{e}^{\text {total }}$ being the sum of the free electron density $n_{e}$ and the bound electron density $n_{\text {be }}$.

For interacting with the theta-pinch plasma, a pulsed ${ }^{48} \mathrm{Ca}^{10+}$ beam with a velocity of $3.652 \mathrm{MeV} / \mathrm{u}$ and a $108 \mathrm{MHz}$ repetition rate is provided by the GSI UNILAC accelerator. A fast diamond detector, installed at a distance of $6.0 \mathrm{~m}$ after the plasma column center, is used to register the beam pulse signal. The measurement of the total energy loss is carried out by means of the differential time of flight (TOF) method. The $108 \mathrm{MHz}$ clock of the accelerator is used as the reference signal.

The phase difference between the $108 \mathrm{MHz}$ clock and the vacuum-TOF reference signal, originating from the ion beam pulses traveling through the evacuated theta-pinch setup, is measured and noted as $t_{1}$. Analogously, the (time dependent) phase difference between the $108 \mathrm{MHz}$ clock 
and the beam pulse signal from the diamond detector during the ion-plasma interaction is measured as $t_{2}$, which depends on the time after triggering the theta-pinch device $\Delta T$. The time difference $\Delta t=t_{2}-t_{1}$ gives the extra flight time due to the energy loss of the beam ions interacting with the plasma. In the experiments described here, $\Delta T$ is tuned such that the plasma is in its intense pinch condition, as mentioned above.

In order to precisely determine the time difference $\Delta t$, the noisy analog signals have been fitted properly. The $108 \mathrm{MHz}$ clock is fitted by a sine function. Regarding the diamond detector signal, it contains the Gaussian profile of the beam and a two-stage response of the detector. Hence, a convolution of a Gaussian function and a two-stage response function is used to fit the recorded beam signal. Figure 2 shows both reference signals and beam signals for the vacuum condition and the plasma condition. The minimum peak value of the sine function for respective reference signals is set as the time zero. Consequently, the corresponding beam signals, being displayed in the same plot, own the same clock. Taking the deconvoluted mass center to represent the whole pulsed beam, the arriving time for the vacuum condition $t_{1}$ is obtained as $3.506 \mathrm{~ns}$, while $t_{2}$ for the plasma condition is obtained as $4.060 \mathrm{~ns}$. It should be noted here that the peak of the measured signal shifts towards a longer time with respect to the beam mass center because of the relatively slow decay response of the detector. Hence, the extra flight time due to plasma $\Delta t$ is calculated as $0.554 \mathrm{~ns}$. For experiments described here, the average time difference is taken as $\Delta t=0.57 \pm 0.1 \mathrm{~ns}$.

Owing to the small change of the ion energy during the entire process of the beam-plasma interaction, $\Delta E$ can be

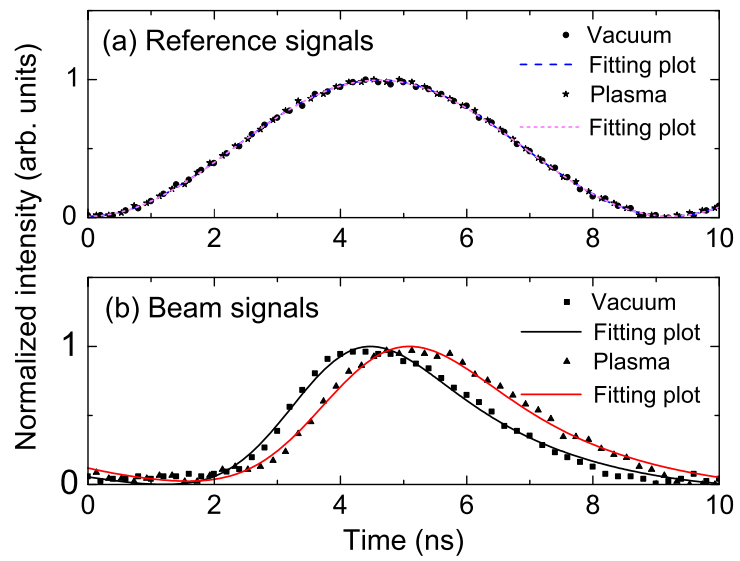

FIG. 2. (a) Reference signals and (b) beam signals for vacuum and plasma conditions. Setting both of the minimum peak values of the respective corresponding reference signals as time zero, the beam signals can be directly compared. Applying the deconvoluted beam mass center as a representative, $t_{1}=3.506 \mathrm{~ns}$ is obtained for the vacuum condition while $t_{2}=4.060$ is obtained for the plasma condition. The time difference $\Delta t$ is obtained as 0.554 ns. linearized with respect to the velocity along the flight path, and hence it is expressed as

$$
\Delta E=2\left(\frac{\Delta t}{T_{0}}\right) E_{0}
$$

where the initial energy $E_{0}$ is given as $175.133 \mathrm{MeV}$ and the flight time for vacuum condition $T_{0}$ is thus calculated as $226.5 \mathrm{~ns}$. Therefore, $\Delta E$ can be easily obtained if only $\Delta t$ is determined. Hence, the total energy loss for our plasma target is obtained as $\Delta E=0.881 \pm 0.155 \mathrm{MeV}$.

In a partially ionized hydrogen plasma target, the swift heavy ion beam loses its energy due to interaction with free and bound electrons. Considering the homogeneity of the plasma target and the (nearly zero) slope of the stopping power function at the ion beam energies, a stepwise integration is not necessary and the total energy loss $\Delta E$ can be expressed as

$$
\Delta E=\left(\left[\frac{d E}{d x}\right]_{\text {free }}+\left[\frac{d E}{d x}\right]_{\text {bound }}\right) \times L
$$

where $L=0.63 \mathrm{~m}$ is the plasma target length, and $[d E / d x]_{\text {free }}$ and $[d E / d x]_{\text {bound }}$ represent the stopping power of the free and bound electrons, respectively.

Taking into account the beam velocity and the free electron temperature of the plasma target, the stopping power of the free electrons is given by the formula $[10,11,14]$

$$
\left[\frac{d E}{d x}\right]_{\text {free }}=\frac{4 \pi n_{e} Z_{\mathrm{eff}}^{2}}{m_{e} v^{2}}\left(\frac{e^{2}}{4 \pi \epsilon_{0}}\right)^{2} \ln \left(\frac{0.764 m_{e} v^{3}}{Z_{\mathrm{eff}} \frac{e^{2}}{4 \pi \epsilon_{0}} w_{p}}\right),
$$

where $Z_{\text {eff }}$ represents the projectile effective charge state, $v$ denotes the projectile velocity, $m_{e}$ and $e$ are the electronic mass and charge, $\epsilon_{0}$ is the vacuum permittivity, and $w_{p}=$ $\sqrt{e^{2} n_{e} \epsilon_{0}^{-1} m_{e}^{-1}}$ is the plasma frequency. The only unknown physical quantity here is the effective charge state. It can be estimated from a charge-state distribution measurement, using a charge-state spectrometer which consists of a dipole magnet, a scintillator, and a scintillation observing camera. The charge-state distributions of the ion beam traveling through the target under cold gas and discharge conditions are shown in Fig. 3.

The initial charge state of the projectiles entering the target is $10+$. For the cold gas condition, the peak charge state is $11+$ and the average is calculated as $11.3+$. The stripping of the projectiles in the cold gas is weak. Under the discharge condition, a peak charge state of $13+$ and an average of $12.7+$ are measured. It should be noted that this distribution is a time-integrated result for a long beam duration which covers the main discharge. During this discharge, while the plasma target is in its intense pinch 


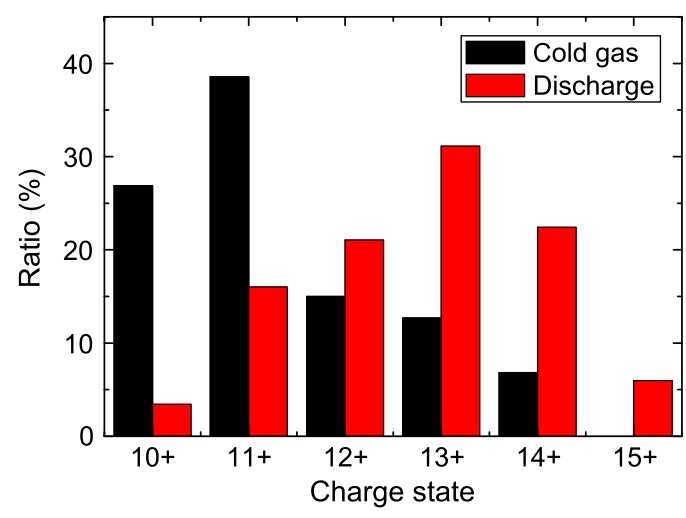

FIG. 3. Charge-state distributions of the beam ions. The effective charge state for the pinch plasma target is estimated as $13 \pm 1$.

phase, the projectiles should be stripped more effectively, and an effective charge state of $13 \pm 1$ is hence considered.

The energy loss due to free electrons is accordingly calculated as $0.177 \pm 0.035 \mathrm{MeV}$, where the uncertainty stems from the effective charge state and the measured free electron density. Using Eq. (2), where the determined total energy loss $\Delta E=0.881 \pm 0.155 \mathrm{MeV}$ and the measured plasma length $L=0.63 \mathrm{~m}$, the energy loss due to bound electrons $[\Delta E]_{\text {bound }}=0.704 \pm 0.190 \mathrm{MeV}$ is obtained, and the corresponding differential stopping power results to $[d E / d x]_{\text {bound }}=1.117 \pm 0.302 \mathrm{MeV} / \mathrm{m}$.

Using the Bethe formula,

$$
\left[\frac{d E}{d x}\right]_{\text {bound }}=\frac{4 \pi n_{\mathrm{be}} Z_{\mathrm{eff}}^{2}}{m_{e} v^{2}}\left(\frac{e^{2}}{4 \pi \epsilon_{0}}\right)^{2} \ln \left(\frac{2 m_{e} v^{2}}{I}\right),
$$

where $I$ represents the ionization potential of $13.6 \mathrm{eV}$ for hydrogen atoms, the unknown bound electron density $n_{\text {be }}$ can be derived. Applying a wide range of charge states from $12+$ to $14+$ as the effective charge state, $n_{\text {be }}$ is calculated as $(1.7 \pm 0.7) \times 10^{17} \mathrm{~cm}^{-3}$. As the free electron density $n_{e}$ is known as $(1.9 \pm 0.1) \times 10^{16} \mathrm{~cm}^{-3}$, the total hydrogen density $n_{0}$, equaling the total electron density $n_{e}^{\text {total }}$, is hence calculated as $(1.9 \pm 0.7) \times 10^{17} \mathrm{~cm}^{-3}$. Furthermore, a low ionization degree which is equivalent to the ratio between the free electron density and the total hydrogen density ranges from $7 \%$ to $17 \%$. With the two known plasma parameters of the free electron density and the ionization degree, the third plasma parameter of the free electron temperature $T_{e}=1.01 \pm 0.04 \mathrm{eV}$ is obtained from FLYCHK with a nonlocal thermodynamic equilibrium plasma condition. As a comparison, a rigorous quantum mechanical method, described in Ref. [31], gives almost the same $T_{e}$ value.

In summary, a novel method to precisely determine the total hydrogen density in a lowly ionized hydrogen plasma, using the energy loss of a swift heavy ion beam, is proposed and realized for the first time. Through measuring the very precisely accessible quantities "free electron density," "charge-state distribution," and "heavy ion energy loss," the total hydrogen density in a pinched hydrogen plasma can be derived. Here, the obtained value of the total hydrogen density for the pinched plasma condition is $(1.9 \pm 0.7) \times 10^{17} \mathrm{~cm}^{-3}$. Compared to the results derived from the measured plasma temperature, the precision of the novel method is increased by about 2 orders of magnitude. Correspondingly, the error of the derived plasma temperature is reduced from $20 \%-30 \%$ to $4 \%$. The successful determination of the total hydrogen density by using a heavy-ion beam opens a new pathway for diagnosing potential WDM. This novel method can also be applied to fully ionized plasmas and cold matter, representing the high and low free electron density limits, respectively.

This work is funded by BMBF (the German Ministry for Education and Science) under Contract No. 05P12RFRB8. The infrastructure of the GSI Helmholtzzentrum für Schwerionenforschung, Darmstadt, was used to do the research presented here. G. X., A. S., and J. W. received funding from HGS-HIRe (the Helmholtz Graduate School for Hadron and Ion Research) for FAIR (the Facility for Antiproton and Ion Research). S.-X. Q. is supported by a postdoctoral research fellowship from the Alexander von Humboldt Foundation. We are very grateful for Dr. Christian Teske's great contribution of manufacturing the spherical theta-pinch device.

\section{"g.xu@gsi.de}

[1] R. P. Dias and I. F. Silvera, Science 355, 715 (2017).

[2] D. Keefe, Annu. Rev. Nucl. Part. Sci. 32, 391 (1982).

[3] R. C. Arnold and J. M. ter Vehn, Rep. Prog. Phys. 50, 559 (1987).

[4] U. Neuner et al., Phys. Rev. Lett. 85, 4518 (2000).

[5] H. Bethe, Ann. Phys. (Berlin) 397, 325 (1930).

[6] L. Northcliffe and R. Schilling, At. Data Nucl. Data Tables 7, 233 (1970).

[7] See http://www.srim.org.

[8] S. Agostinelli et al., Nucl. Instrum. Methods Phys. Res., Sect. A 506, 250 (2003).

[9] C. Deutsch et al., in Proceedings of the International Symposium on Heavy Ion Inertial Fusion, Darmstadt, Germany, 1988 [Nucl. Instrum. Methods Phys. Res., Sect. A 278, 38 (1989)].

[10] K. Weyrich et al., in Proceedings of the International Symposium on Heavy Ion Inertial Fusion, Darmstadt, Germany, 1988 [Nucl. Instrum. Methods Phys. Res., Sect. A 278, 52 (1989)].

[11] D. H. H. Hoffmann, K. Weyrich, H. Wahl, D. Gardés, R. Bimbot, and C. Fleurier, Phys. Rev. A 42, 2313 (1990).

[12] G. D. Alton, R. A. Sparrow, and R. E. Olson, Phys. Rev. A 45, 5957 (1992).

[13] K.-G. Dietrich, D. H. H. Hoffmann, E. Boggasch, J. Jacoby, H. Wahl, M. Elfers, C. R. Haas, V.P. Dubenkov, and A. A. Golubev, Phys. Rev. Lett. 69, 3623 (1992). 
[14] D. Hoffmann et al., Nucl. Instrum. Methods Phys. Res., Sect. B 90, 1 (1994).

[15] J. Jacoby et al., Phys. Rev. Lett. 74, 1550 (1995).

[16] C. Teske and J. Jacoby, IEEE Trans. Plasma Sci. 36, 1930 (2008).

[17] C. Teske, J. Jacoby, W. Schweizer, and J. Wiechula, Rev. Sci. Instrum. 80, 034702 (2009).

[18] C. Teske, B.-J. Lee, A. Fedjuschenko, J. Jacoby, and W. Schweizer, IEEE Trans. Plasma Sci. 38, 1675 (2010).

[19] C. Teske, J. Jacoby, F. Senzel, and W. Schweizer, Phys. Plasmas 17, 043501 (2010).

[20] C. Teske, Y. Liu, S. Blaes, and J. Jacoby, Phys. Plasmas 19, 033505 (2012).

[21] G. Loisch et al., in Proceedings of the 19th IEEE Pulsed Power Conference (PPC), San Francisco, 2013 (IEEE, New York, 2013), p. 1.

[22] G. Loisch et al., IEEE Trans. Plasma Sci. 42, 1163 (2014).
[23] G. Loisch, G. Xu, A. Blazevic, B. Cihodariu-Ionita, and J. Jacoby, Phys. Plasmas 22, 053502 (2015).

[24] G. Xu, C. Hock, G. Loisch, G. Xiao, J. Jacoby, K. Weyrich, Y. Li, and Y. Zhao,, Phys. Plasmas 22, 052703 (2015).

[25] H. R. Griem, Plasma Spectroscopy (McGraw-Hill, New York, 1964).

[26] M. Ivkovi, N. Konjevi, and Z. Pavlovi, J. Quant. Spectrosc. Radiat. Transfer 154, 1 (2015).

[27] H.-K. Chung, W. Morgan, and R. Lee, J. Quant. Spectrosc. Radiat. Transfer 81, 107 (2003).

[28] H.-K. Chung, M. Chen, W. Morgan, Y. Ralchenko, and R. Lee, High Energy Density Phys. 1, 3 (2005).

[29] I. Langmuir, J. Am. Chem. Soc. 37, 417 (1915).

[30] D. Mihalas, W. Dappen, and D. G. Hummer, Astrophys. J. 331, 815 (1988).

[31] O. Theimer and P. Kepple, Phys. Rev. A 1, 957 (1970). 